\title{
Unintended retention of a ruptured radiopaque thread extending from the corner of a gauze during laparoscopy
}

\author{
Yoshiaki Oshima $^{1 *}$ D, Osamu Yamamoto ${ }^{2}$, Akihiro Otsuki ${ }^{3}$, Saori Tokunaga', Keiichiro Ueda ${ }^{1}$ and Yoshimi Inagaki ${ }^{3}$
}

\begin{abstract}
Small gauze is used in laparoscopy; therefore, retention of gauze can occur. We experienced a case of retention of a radiopaque thread that ruptured from a piece of gauze and moved into the peritoneum during a scheduled laparoscopy. The patient was a 65-year-old woman who underwent laparoscopic-assisted transverse colon resection for transverse colon cancer. A commercial gauze commonly used for laparoscopy was used during the surgery. To more easily identify the gauze during surgery, radiopaque threads extending up to $3.0 \mathrm{~cm}$ from the two diagonal corners of the gauze body were attached. After wound closure, radiography showed a radiopaque thread-like substance in the abdomen. Minor laparotomy was performed, and part of the radiopaque thread was discovered. On postoperative day 22, the patient was in remission and discharged.
\end{abstract}

Keywords: Laparoscopy, Retention of gauze, Radiopaque thread, Gauze count, Electric scalpel

\section{Letter to the Editor}

The frequency of unintended retention of gauze during surgery is 1 in 8300 cases [1], and the abdomen and pelvis are the most common anatomic sites of retention, accounting for $50.2 \%$ of such occurrences [2]. In patients with unintended retention of gauze, the gauze count was judged as correct in $86 \%$ of the cases [2]. Small gauze is used in laparoscopy, and therefore, unintentional retention of gauze can occur. The frequency of unintended retention of gauze has not yet been reported. We experienced a case of retention of a radiopaque thread that ruptured from the corner of a gauze and moved into the peritoneum during a scheduled laparoscopy.

The patient was a 65 -year-old woman with a height of $151 \mathrm{~cm}$ and weight of $39 \mathrm{~kg}$. At the age of 53, she underwent laparoscopic-assisted transverse colon resection due to transverse colon cancer. Surgery time was $3 \mathrm{~h} 18 \mathrm{~min}$, and the volume of perioperative blood loss was $15 \mathrm{ml}$. Gauze count was performed four times: at the time of preparing the surgical

\footnotetext{
*Correspondence: yoshimatomo@gmail.com

${ }^{1}$ Department of Anesthesiology, Yonago Medical Center, 4-17-1 Kuzumo,

Yonago, Tottori 683-0006, Japan

Full list of author information is available at the end of the article
}

instruments, immediately before the start of surgery, at the start of wound closure, and at the time of skin suture. At all times, the gauze count was found to be correct. The surgery was completed uneventfully. After wound closure, abdominal radiography was performed, and a radiopaque thread-like substance was found in the left side of the abdomen (Fig. 1). When all pieces of gauze used in the procedure were examined, one was found to have a radiopaque thread that had shortened due to rupture (Fig. 2a, bottom). The gauze itself had some burn marks on it (Fig. 2b); the end of the thread also had evidence of being burnt by an electric scalpel (Fig. 2c). Although it seemed that only the thread that ruptured from the corner of the gauze entered the peritoneum, the possibility of retention of the gauze itself could not be completely ruled out. Minor laparotomy was performed to look for the retained object. The part of the radiopaque thread that had ruptured from the gauze was discovered under fluoroscopic guidance (Fig. 2c), confirming that the gauze itself was absent during wound closure. On postoperative day 22, the patient was in remission and discharged. She has progressed uneventfully till the time of writing this correspondence.

(C) The Author(s). 2019 Open Access This article is distributed under the terms of the Creative Commons Attribution 4.0 International License (http://creativecommons.org/licenses/by/4.0/), which permits unrestricted use, distribution, and reproduction in any medium, provided you give appropriate credit to the original author(s) and the source, provide a link to the Creative Commons license, and indicate if changes were made. The Creative Commons Public Domain Dedication waiver (http://creativecommons.org/publicdomain/zero/1.0/) applies to the data made available in this article, unless otherwise stated. 


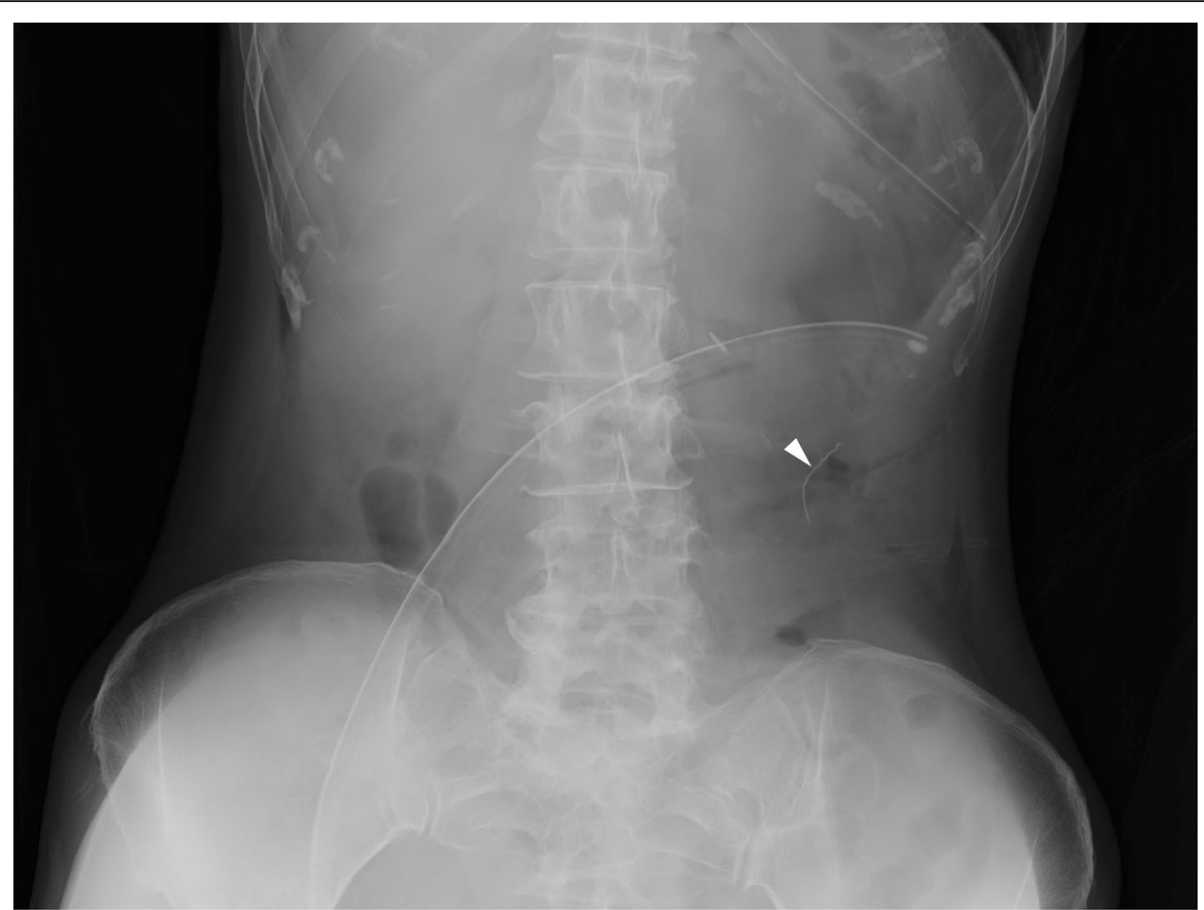

Fig. 1 An X-ray image of the abdomen showed a radiopaque thread-like substance retained in the left part of the abdomen (arrowhead)
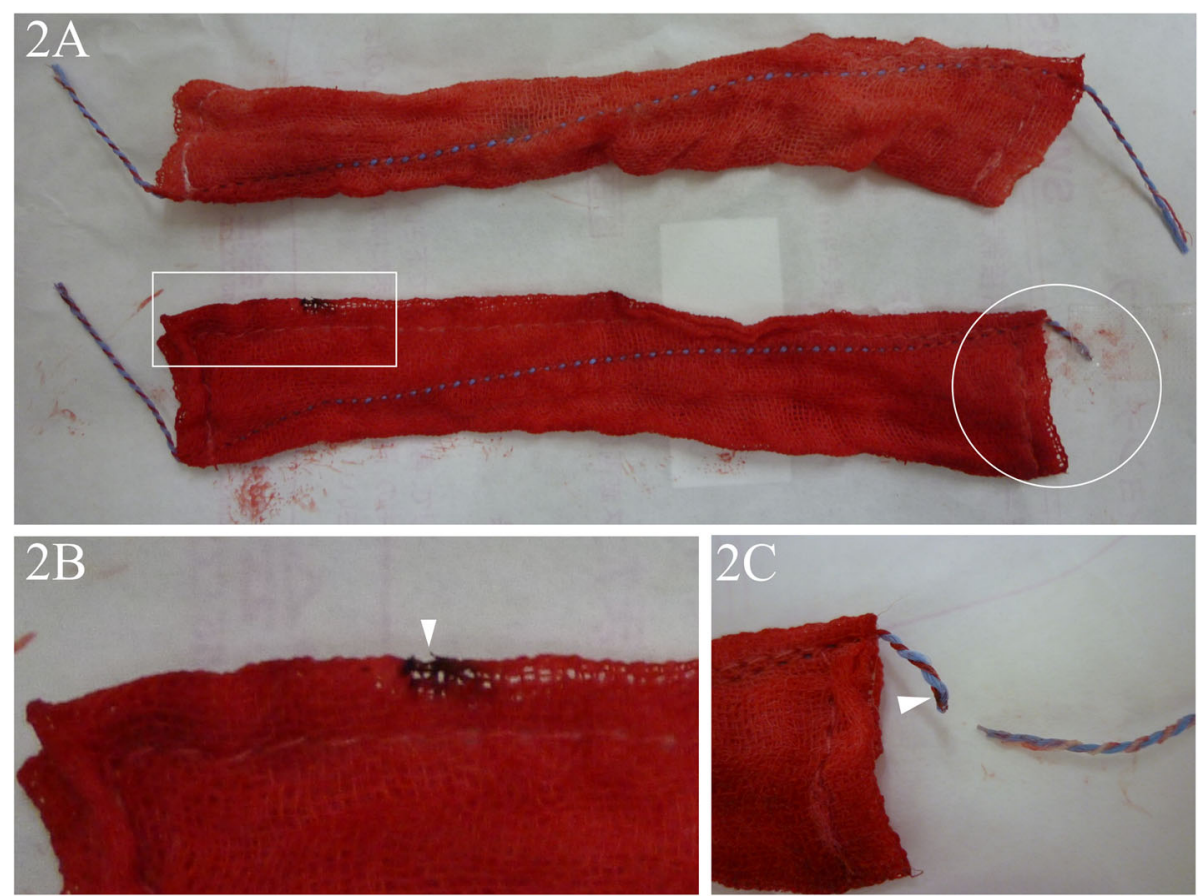

Fig. 2 a. The upper panel shows regular gauze with the extending threads intact after its use. The lower panel shows gauze from which the extending thread ruptured at its midpoint during its use. $\mathbf{b}$. A portion of the gauze body (enlarged image of the area in the white rectangle in $\mathbf{a}$ ) has a burnt mark (arrowhead). c. The end of the thread remaining on the gauze (enlarged image of the area in the white circle in $\mathbf{a}$ ) and the portion of the thread that entered the peritoneum are shown. The end of the remnant thread extending from the corner of the gauze has a burnt mark (arrowhead) 
The gauze (Trox, Osaki Medical, Nagoya, Japan) used during surgery has been commercialised for laparoscopy. The body of the gauze is made of cotton; it is thin and long, with dimensions of $3.0 \times 15.0 \mathrm{~cm}$ to enable its passage through a port (Fig. 2). As the lumen of the port is narrow, the corners of the gauze are compressed between forceps; the long axis of the gauze and that of the port are matched, and the gauze is then pulled out from the peritoneum through the port. For easy identification of the gauze during surgery and easily identifying the corners of the gauze, radiopaque threads extending up to $3.0 \mathrm{~cm}$ from the two diagonal corners of the gauze body are attached (Fig. 2a, top). A similar radiopaque thread is sewn diagonally through the gauze body (Fig. 2 ). The thread is made of polypropylene and polyester and becomes radiopaque when soaked in barium sulphate. Polypropylene and polyester per se are inactive. The maker put a precaution in the catalogue that direct contact of the extending radiopaque thread with an electric scalpel or direct compression with forceps should be avoided when using the gauze. The maker also produces gauze (Trox II) of the same size that has no radiopaque extension thread. We are presently using Trox II in laparoscopy at our hospital.

\section{Acknowledgements}

We would like to thank Editage (www.editage.com) for English language editing.

\section{Authors' contributions}

YO - analysis and interpretation of data, drafting the manuscript; OY acquisition of data, revising the manuscript; $\mathrm{AO}$ - conception of the work, revising the manuscript; ST - acquisition of data, revising the manuscript; $\mathrm{KU}$ - acquisition of data, revising the manuscript; Yl - conception of the work, revising the manuscript. All authors read and approved the final manuscript.

Funding

Not applicable.

Availability of data and materials

The datasets used and/or analysed during the current study are available from the corresponding author on reasonable request.

Ethics approval and consent to participate

Not applicable.

Consent for publication

The patient gave written informed consent for publication of this article.

\section{Competing interests}

The authors declare that they have no competing interests.

\section{Author details}

'Department of Anesthesiology, Yonago Medical Center, 4-17-1 Kuzumo, Yonago, Tottori 683-0006, Japan. 'Department of Gastrointestinal Surgery, Yonago Medical Center, 4-17-1 Kuzumo, Yonago, Tottori 683-0006, Japan. ${ }^{3}$ Division of Anesthesiology and Critical Care Medicine, Department of Surgery, Tottori University Faculty of Medicine, 36-1 Nishi-cho, Yonago, Tottori 683-8504, Japan.
Received: 15 August 2019 Accepted: 26 August 2019

Published online: 04 September 2019

References

1. Cima RR, Kollengode A, Garnatz J, Storsveen A, Weisbrod C, Deschamps C. Incidence and characteristics of potential and actual retained foreign object events in surgical patients. J Am Coll Surg. 2008;207:80-7.

2. Steelman VM, Shaw C, Shine L, Hardy-Fairbanks AJ. Retained surgical sponges: a descriptive study of 319 occurrences and contributing factors from 2012 to 2017. Patient Saf Surg. 2018;12:20.

\section{Publisher's Note}

Springer Nature remains neutral with regard to jurisdictional claims in published maps and institutional affiliations. 